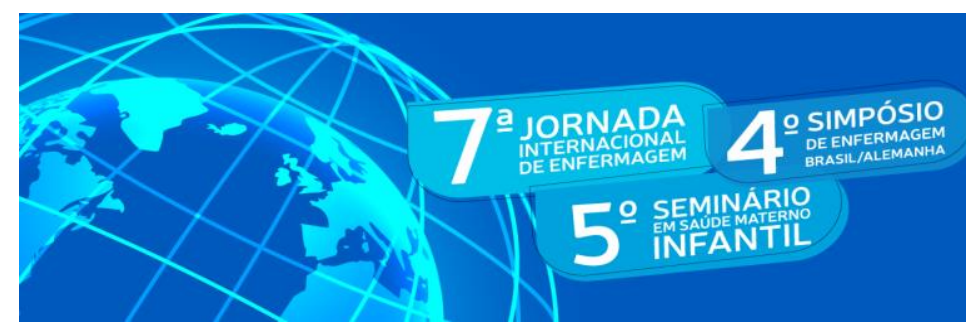

PRÁTICA AVANÇADA EM SAÚdE:

desafios e projeçōes interprofissionais

05 a 07.05 de 2021

ISSN: 2318-0234

DOI: http://doi.org/10.48195/jie2021-185

\title{
MANEJO DO DESENVOLVIMENTO A PARTIR DOS REGISTROS PROFISSIONAIS NA CADERNETA DE SAÚDE DA CRIANÇA ${ }^{1}$
}

\section{Flávia Baluz Bezerra de Farias Nunes ${ }^{2}$; Francisca Georgina Macedo de Sousa ${ }^{3}$; Jéssica Nathália de Melo Sousa ${ }^{4}$; Joyce Karla Serra Santos ${ }^{5}$; Luana Câmara da Silva ${ }^{6}$;}

\section{RESUMO}

Objetivo: Avaliar o registro dos marcos do desenvolvimento infantil realizado pelos profissionais da Atenção Primária em Saúde na Caderneta de Saúde da Criança. Método: estudo descritivo documental quantitativo realizado em 25 UBS's de São Luís-MA cujo número de consultas pediátricas foi superior a 1000 em 2018. A coleta de dados ocorreu entre maio e setembro de 2019. Analisaram-se os registros no instrumento de vigilância do desenvolvimento (IVD) de 1.736 CSC com distribuição estratificada por unidade. Resultados: do total de Cadernetas de Saúde da Criança avaliadas, em 70,5\% o IVD não foi registrado quando das consultas na Atenção Primária em Saúde. Nas Cadernetas de crianças na faixa etária de 1 a 12 meses, $84,5 \%$ não havia registro de nenhum marco do desenvolviemnto e em e em $14 \%$ foi registrado adequadamente. Quanto ao IVD de crianças de 1 a 3 anos, 94\% das CSC's não apresentaram registro e 5,5\% foram preenchidos corretamente. Conclusão: Mediante a ausência de registros para acompanhar o desenvolvimento, eixo base da avaliação da saúde infantil, infere-se que é necessário reforçar com profissionais e familiares a importância dos registros na CSC.

Palavras-chave: Comunidade; Enfermagem; Família.

\begin{abstract}
Purpose: To evaluate the registration of the development milestones by the professionals of the Primary Attention in Health in the Child Health Report. Method: documental descriptive study with quantitative approach accomplished in 25 UBS's from São Luís - MA which numbers of pediatric consultations were over 1000 in 2018. The data collection occurred from May to September in 2019. The registration were analyzed in the development surveillance instrument (IDV) of 1.736 CSC with stratified distribution for each unit. Results: Of the total of the Child Health Reports evaluated, in 70,5\% the IVD was not registered in the Primary

\footnotetext{
${ }^{1}$ Parte do TCC do Curso de Graduação em Enfermagem da Universida de Federal do Maranhão.

${ }^{2}$ Enfermeira Doutora em Ciências pela USP. Docente UFMA. Email: flavia.farias@ufma.br

${ }^{3}$ Enfermeira. Orientadora. Pós-doutora em Enfermagem. Docente UFMA. Email: georginamacedo17@gmail.com

${ }^{4}$ Enfermeira Residente em Saúde da Criança.HUUFMA. Membro do Grupo de Ensino e Pesquisa na Saúde da Família, Criança e Adolescente - GEPSFCAEmail: jessica-nathy@hotmail.com

5 Enfermeira do Instituto de Apoio à Vida Humana, São Luís-MA. Email: jkarla serra @gmail.com

${ }^{6}$ Enfermeira do Hospital Clementino Moura, São Luís-MA. Email: lucamaradasilva @ gmail.com
} 


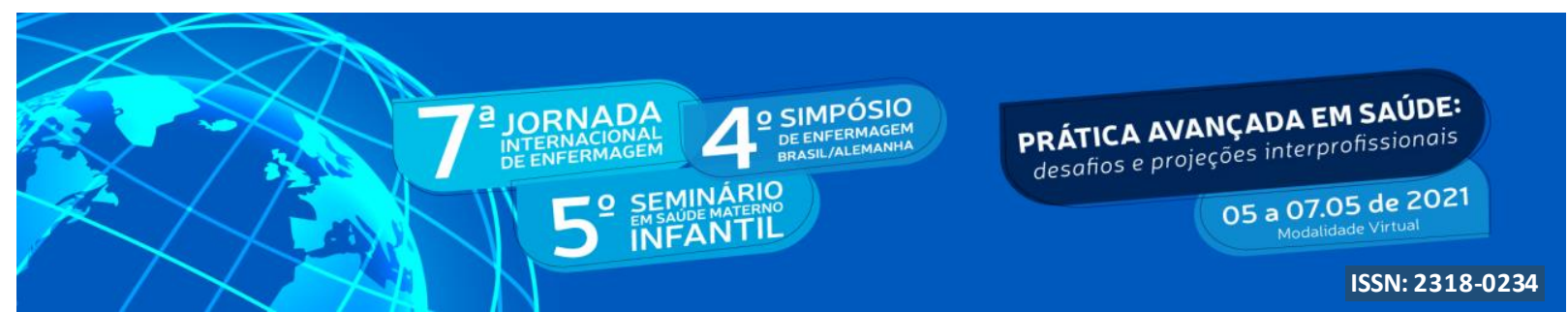

Attention in Health. In the children's reports, the age group from 1 to 12 months, $84,5 \%$ there was no registration of any development milestone and $14 \%$ were fill in correctly. In addition, about the IVD from 1 to 3 years, 94\% of the CSC's did not present registration and 5,5\% were fill in correctly. Conclusion: Through the absence of registration to monitor the development, basic pillar of the evaluation of the child health, it is possible to infer that is necessary to reinforce to the professionals and families the importance of the registration in the CSC.

Key Words: Community; Nursing; Family.

\section{INTRODUÇÃO}

Em 1984, foi criado o Programa de Assistência Integral à Saúde da Criança (PAISC) cujas principais ações foram direcionadas ao monitoramento do crescimento e do desenvolvimento, controle de diarreias, desidratação e infecções respiratórias agudas, prevenção e manejo do recém-nascido de baixo peso, prevenção de acidentes e intoxicações e assistência ao recém-nascido (BRASIL, 2011).

De modo geral, entre as décadas de 1980 e 1990, houve muitas conquistas no âmbito da saúde, ao trazer a saúde como um direito, que necessita de abordagem integral. Essa tendência se fortaleceu na assistência infantil com a criação da Caderneta de Saúde da Criança (CSC) (BRASIL, 2005a; SANINE et al., 2018). Nesse cenário, a CSC torna-se um instrumento facilitador do acompanhamento integral da saúde da criança, tendo em vista que ela é um documento mais completo que o Cartão da Criança, com o objetivo de aprimorar a vigilância à saúde da criança desde o nascimento (BRASIL, 2005a).

Dentre as ações de saúde infantil, destaca-se a avaliação do desenvolvimento, que é um dos componentes fundamentais para a vigilância à saúde da criança. Vale ressaltar, que essa avaliação deve fazer parte da consulta geral da criança, não necessita de equipamentos especializados ou espaço específico para ocorrer, embora alguns brinquedos ou objetos presentes no consultório possam ser utilizados para desencadear reflexos ou alguns marcos do desenvolvimento (BRASIL, 2012).

Além disso, embora os marcos tradicionais sejam os instrumentos guia para monitorar o desenvolvimento, sua análise requer sensibilidade, tendo em vista que focar em um marco pontual pode prejudicar a capacidade de avaliar o desenvolvimento da criança como um todo (BRASIL, 2002). 


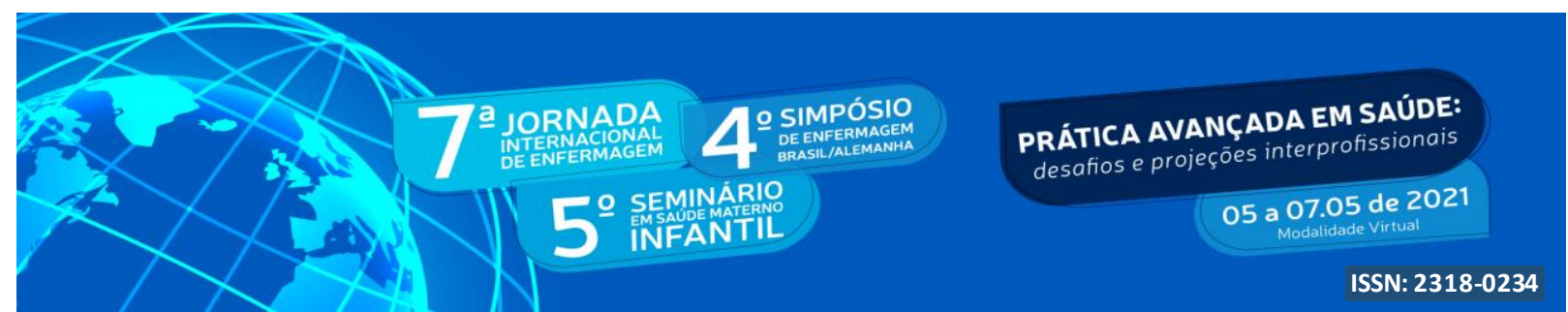

Diante da complexidade que permeia o desenvolvimento infantil, entende-se que sua avaliação não deve ser limitada a procedimentos técnicos isolados, tendo em vista que está relacionado a um conjunto de fatores ambientais, sociais e econômicos. Portanto, o profissional de saúde deve investigar esses determinantes na tentativa de realizar um diagnóstico situacional da família da criança e do meio onde essa se insere e direcionar os cuidados previstos (MONTEIRO et al., 2014).

Partindo do exposto, entende-se que a CSC tem a finalidade de reunir as informações de saúde essenciais em um único instrumento, permitindo que os profissionais que atuam em diferentes espaços assistenciais às crianças tenham acesso a essas informações. Nesse sentido, reforça-se que a qualidade desses registros é um dos alicerces da vigilância do desenvolvimento infantil.

Quando das aulas práticas da disciplina Saúde da Criança e do Adolescente, fui aos poucos identificando que as informações da saúde da criança a partir das ações definidas na Política de Atenção Integral a Saúde da Criança - PNAISC (BRASIL, 2015), não estavam registradas na CSC, ou quando existentes, não atendiam às recomendações para o registro das mesmas. Esse apontamento foi mobilizador para investigar: Como os profissionais de saúde registram os marcos do desenvolvimento infantil na CSC? Como se caracterizam esses registros?

Desse modo, torna-se relevante realizar um estudo acerca do manejo da Caderneta de Saúde da Criança pelos profissionais de saúde, com relação ao acompanhamento do desenvolvimento, com o intuito de qualificar a atuação dos profissionais, pois é por meio do registro adequado das informações da criança que é realizada a vigilância em saúde infantil eficaz.

\section{OBJETIVO}

Avaliar os marcos do desenvolvimento infantil a partir dos registros profissionais realizados na Caderneta de Saúde da Criança. 


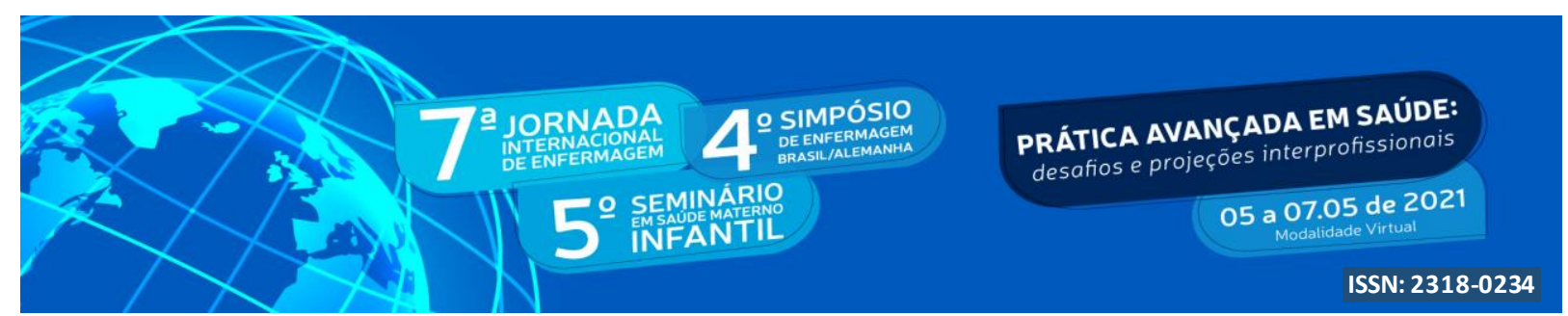

\section{METODOLOGIA}

Esse estudo faz parte de uma pesquisa maior, intitulada "Caderneta de Saúde da Criança e o manejo profissional das ações de saúde infantill”, cujo objetivo é avaliar como as ações de saúde da criança são registradas na CSC. Trata-se de estudo descritivo, do tipo documental com abordagem quantitativa a partir dos registros realizados pelos profissionais na Caderneta de Saúde da Criança.

A coleta de dados se deu em 25 UBS's do município de São Luís-MA, no perído de maio a outubro de 2019, em que foram coletadas 1.736 CSCs, mediante visitas às UBS's, tendo como contexto primário para a coleta a sala de vacinação, onde há maior fluxo de crianças. As CSCs foram solicitadas à vacinadora e escaneadas enquanto a criança era vacinada. Esse processo reduziu o tempo de coleta de dados e permitiu a análise dos registros por meio do instrumento de coleta de dados.

Com o intuito de operacionalizar a análise do manejo das CSC foi elaborado um instrumento composto por 14 dimensões e 98 itens, que englobou todas as informações contidas na $7^{\mathrm{a}}$ edição da Caderneta de Saúde da Criança. Para fins desta pesquisa, foram considerados os itens do Instrumento de Vigilância do Desenvolvimento, sendo assim foram analisados 3.472 IVDs, tendo em vista que cada CSC apresenta dois instrumentos, um de 1 a 12 meses e outro para 1 a 3 anos

O processamento dos dados foi realizado por uma equipe de digitadores utilizando o programa Microsoft Excel 2016 e utilizado o programa Stata 12.0 (Stata Corp, College Station, United States) para análise estatística descritiva dos dados coletados.

O preenchimento dos itens de cada dimensão foi avaliado de acordo com as orientações do Manual para Utilização da Caderneta de Saúde da Criança, como correto, incorreto ou ausente (BRASIL, 2005b). Considerou-se registro correto os que estavam de acordo com as recomendações do Ministério da Saúde. Com relação ao Instrumento de Vigilância do Desenvolvimento, deveria estar preenchido com P, A e NV para ser considerado correto, qualquer outra forma de registro foi considerada incorreta. Além disso, também foi avaliada a quantidade de registros para a idade, caracterizand o o preenchimento como completo e incompleto, ao compará-lo com o calendário mínimo de consultas definido 


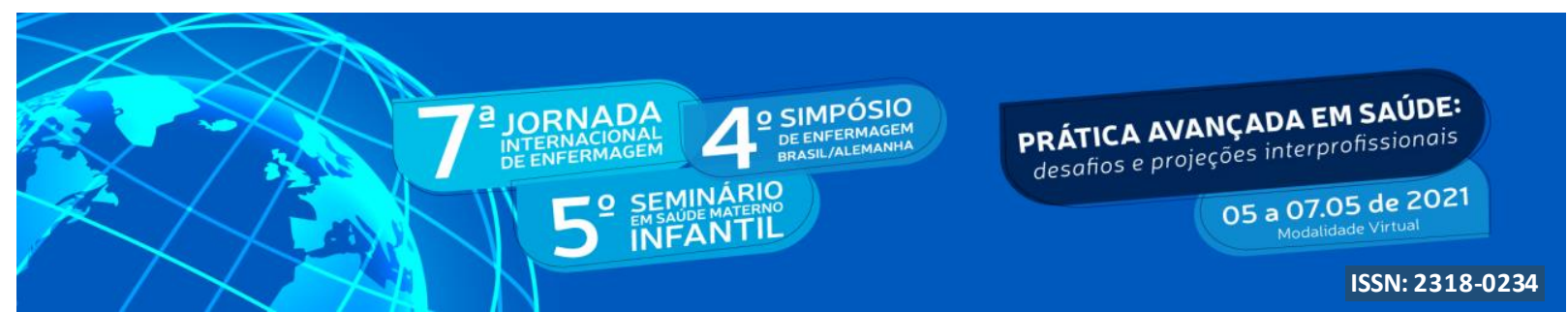

pelo Ministério da Saúde (2012). Vale ressaltar que considerou-se as CSC's cuja criança estava na faixa etária indicada para ter aquele registro. Nesse sentido, quanto ao manejo do Instrumento de Vigilância do Desenvolvimento de 1 a 12 meses, foram excluídas dessa análise as CSC's de crianças com idade inferior a 1 mês. Para a análise do preenchimento dos marcos do desenvolvimento >12 meses a 3 anos, foram excluídas as CSC's de crianças com 12 meses ou menos. Vale ressaltar que para crianças acima de 12 meses, avaliou-se o preenchimento de ambos Instrumentos de Vigilância do Desenvolvimento.

Embora trate-se de pesquisa documental, em que foi avaliado o preenchimento da Caderneta de Saúde da Criança, a Resolução nº 510 (BRASIL, 2016), estabelece que estas pesquisas, quando para os Trabalhos de Conclusão de Curso, monografias e similares, devem apresentar o protocolo de pesquisa ao sistema Comitê de Ética em Pesquisa/Conselho

Nacional de Ética em Pesquisa. Sendo assim, a pesquisa em questão teve protocolo submetido na Plataforma Brasil com CAAE no 17772819.1.0000.5087, sendo aprovada com parecer $\mathrm{n}^{\circ} 3.785 .268$.

\section{RESULTADOS E DISCUSSÃO}

De forma a facilitar a análise do manejo dos indicadores de crescimento e do desenvolvimento nos registros realizados pelos profissionais de saúde na CSC, optou-se por estratificar a amostra em quatro (04) faixas etárias (Tabela 1).

Tabela 1. Idade das crianças segundo registro na CSC. São Luís, 2019.

\begin{tabular}{ccc}
\hline Faixa etária & $\mathbf{n}$ & $\%$ \\
\hline < 1 mês & 52 & 3,0 \\
1 mês a 12 meses & 595 & 34,3 \\
$>$ 12 meses a 3 anos & 510 & 29,4 \\
$>$ 3 anos & 574 & 33 \\
Sem registro da data de nascimento & 05 & 0,3 \\
\hline Total & 1.736 & 100
\end{tabular}

Fonte: Própria autora, 2019.

A faixa etária mais frequente foi de 1 mês a 1 ano, com n=595 (34,3\%) e a de menor 


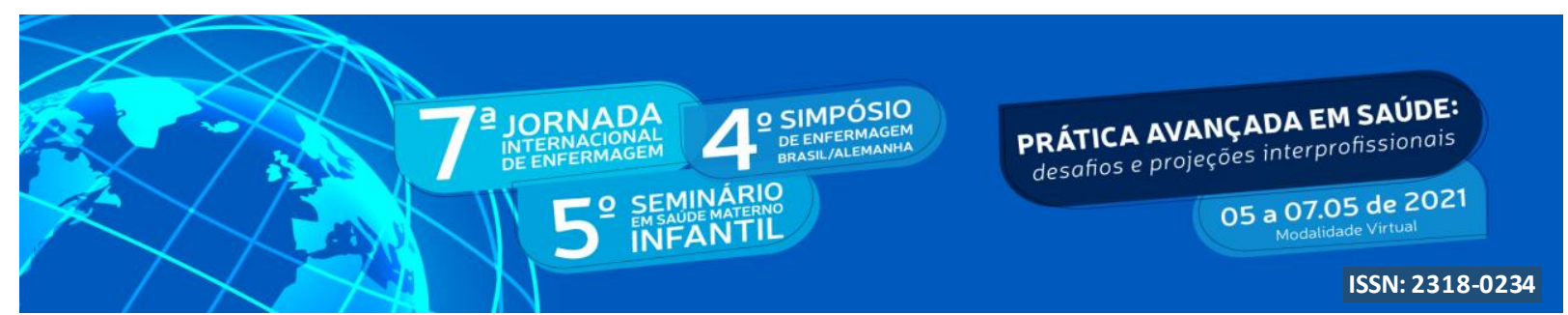

frequência foi menor que 1 mês, com $n=52(3,0 \%)$. Apenas cinco $(0,3 \%)$ CSC não tiveram a idade avaliada, devido à ausência da data de nascimento.

Tabela 2. Presença dos registros no Instrumento de Vigilância do Desenvolvimento da CSC. São Luís, 2019.

Presença dos registros no Instrumentos de Vigilância do Desenvolvimento da CSC

\begin{tabular}{cccccccc}
\hline \multicolumn{2}{c}{ Registro presente } & \multicolumn{2}{c}{ Registro ausente } & \multicolumn{2}{c}{ Não se aplica } & \multicolumn{2}{c}{ Total } \\
\hline $\mathbf{n}$ & $\boldsymbol{\%}$ & $\mathbf{n}$ & $\boldsymbol{\%}$ & $\mathbf{n}$ & $\boldsymbol{\%}$ & $\mathbf{n}$ & $\boldsymbol{\%}$ \\
325 & 9,4 & 2.448 & 70,5 & 699 & 20,1 & 3.472 & 100 \\
\hline
\end{tabular}

Fonte: Própria autora, 2019.

$\mathrm{Na}$ Tabela 2, observa-se o panorama geral dos Instrumentos de Vigilância do Desenvolvimento analisados $(n=3.472)$, em que 70,5\% dos IVDs $(n=2.448)$ não apresentaram registro da avaliaçãodo desenvolvimento.

Tabela 3. Presença do Registro dos Marcos do Desenvolvimento na CSC por faixa etária. São Luís, 2019.

\begin{tabular}{ccccccc}
\hline \multirow{2}{*}{$\begin{array}{c}\text { Instrumento de } \\
\text { Vigilância }\end{array}$} & \multicolumn{2}{c}{ Presença dos Registros dos Marcos do Desenvolvimento na CSC } \\
\cline { 2 - 7 } & \multicolumn{2}{c}{ Registro presente } & Sem registro & \multicolumn{2}{c}{ Total } \\
\hline & $\mathbf{n}$ & $\%$ & $\mathbf{n}$ & $\%$ & $\mathbf{n}$ & $\%$ \\
$\mathbf{1}$ a 12 meses & 261 & 15,5 & 1.423 & 84,5 & 1.684 & 100 \\
$>\mathbf{1 2}$ meses a 3 anos & 64 & 5,8 & 1.025 & 94,0 & 1.089 & 100 \\
\hline
\end{tabular}

Fonte: Própria autora, 2019.

A Tabela 3 traz os dados referentes à presença dos registros dos marcos do desenvolvimento por faixa etária, sendo identificado ausência de registros em 84,5\% das CSC no Instrumento de Vigilância do Desenvolvimento (IVD) de 1 a 12 meses e 94,0\% nas cadernetas de crianças de 12 meses a 3 anos. 


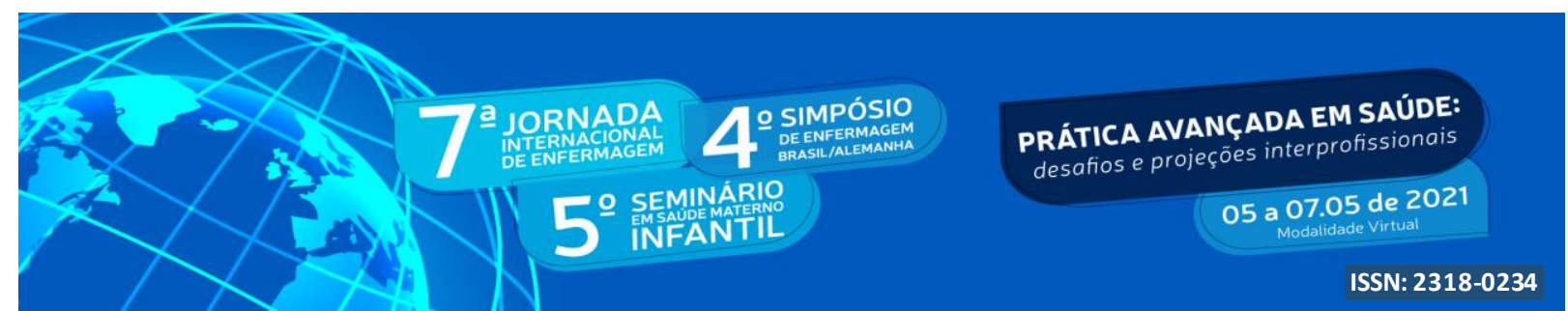

Tabela 4. Tipificação do Registro dos Marcos do Desenvolvimento na CSC por faixa etária. São Luís, 2019.

\begin{tabular}{lcccccc}
\hline \multirow{2}{*}{$\begin{array}{c}\text { Instrumento de } \\
\text { Vigilância }\end{array}$} & \multicolumn{2}{c}{ Tipificação dos Registros dos Marcos do Desenvolvimento na CSC } \\
\cline { 2 - 7 } & \multicolumn{2}{c}{ Correto } & \multicolumn{2}{c}{ Incorreto } & \multicolumn{2}{c}{ Total } \\
\hline \multirow{2}{*}{$\mathbf{1}$ a 12 meses } & $\mathbf{n}$ & $\boldsymbol{\%}$ & $\mathbf{n}$ & $\boldsymbol{\%}$ & $\mathbf{n}$ & $\%$ \\
$\mathbf{1}$ & 237 & 90,0 & 24 & 1,5 & 261 & 100 \\
\hline
\end{tabular}

Fonte: Própria autora, 2019.

No que diz respeito à Tabela 4, apresenta a tipificação desses registros, sendo evidenciado preenchimento correto em 90\% dos IVDs de 1 a 12meses que possuíam essa informação( $n=237$ ), semelhante ao IVD de 1 a 3 anos, em que foi evidenciado preenchimento correto em 92,2\% dos IVDs preenchidos(n=59).

O acompanhamento do crescimento e do desenvolvimento é de suma importância para a promoção da saúde e prevenção de agravos, sendo a CSC o documento proposto pelo Ministério da Saúde para registro desse acompanhamento (BRASIL, 2005b). Vale ressaltar, que para um acompanhamento significativo, é necessário que os profissionais realizem o registro adequado das informações de saúde em cada contato da criança com o serviço (AMORIM et al., 2018)

No entanto, várias pesquisas direcionadas à utilização da CSC apontam deficiências no preenchimento (ALMEIDA et al, 2015; ABUD; GÁIVA, 2015; SILVA; GÁIVA, 2015; AMORIM et al, 2018), o que corrobora com os resultados deste estudo. No que diz respeito ao manejo do Desenvolvimento, a maioria das CSCs avaliadas não apresentava registro, com destaque aos marcos de 12 meses a 3 anos, ausentes em 94,0\% das CSCs.

Resultado semelhante foi encontrado por Abud e Gáiva (2015) em estudo realizado na cidade de Cuiabá, em que 95\% das CSCs avaliadas não apresentavam registro referente ao desenvolvimento neuropsicomotor. Assim como Amorim e colaboradores (2018), que avaliaram a qualidade de preenchimento da CSC, e mesmo nas que apresentavam preenchimento satisfatório, havia baixa frequência de registros do desenvolvimento.

É valido pontuar, que neste estudo foi avaliado o preenchimento de acordo com a faixa 


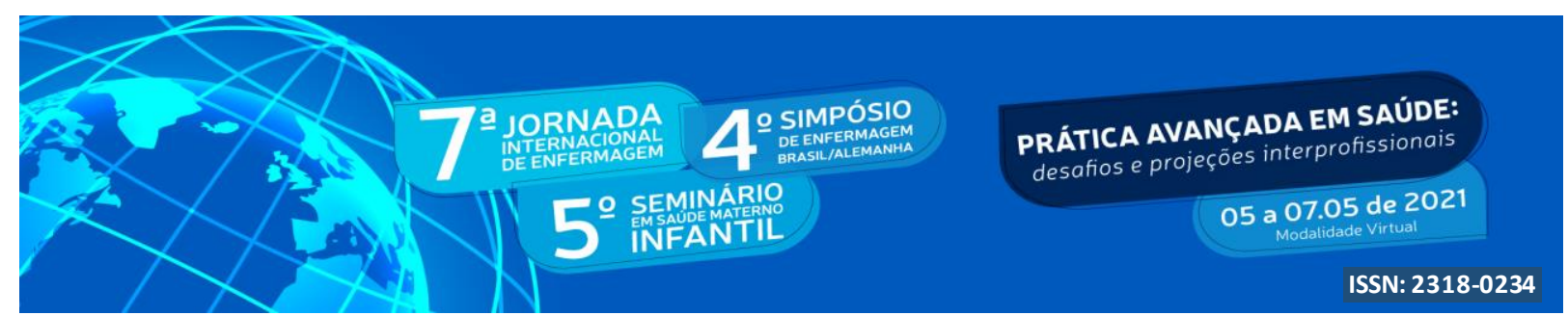

etária, como é dividido na CSC, e obteve-se uma frequência menor de registros ausentes para os marcos de 1 a 12 meses (84,5\%), o que sugere maior preocupação dos profissionais em avaliar o desenvolvimento das crianças menores de um ano. Embora o percentual de preenchimento para ambas as faixas etárias tenha sido baixo.

Em seu estudo, Silva e Gáiva (2015) entrevistaram profissionais da saúde que trabalhavam na ABS e a maioria ressaltou a importância dos registros na CSC para o acompanhamento da saúde infantil. No entanto, com relação à avaliação do desenvolvimento, foi relatada como algo mais observacional sem avaliação específica dos marcos e difícil de realizar apenas com a entrevista do acompanhante. Além disso, pesquisas revelam que quando os profissionais de saúde manuseiam a CSC se direcionam à vacinação e aos gráficos dos indicadores de crescimento, dando especial atenção ao ganho de peso (ANDRADE et al, 2014; SILVA;GÁIVA, 2015)

Nesse sentido, infere-se que a monitoração do desenvolvimento ainda é bastante negligenciada, seja pela priorização de outros aspectos que permeiam a saúde infantil, seja pelo preparo insuficiente para realizá-la.

\section{CONCLUSÃO}

Mediante a realização dessa pesquisa foi possível identificar o manejo inadequado da CSC, que se contrapõe às recomendações da PNAISC, em especial naquilo que diz respeito ao eixo central da atenção à criança, que é a avaliação sistemática do crescimento e do desenvolvimento.

Os registros incorretos sugerem desconhecimento quanto ao modo de utilização desse documento, o que somado à alta frequência de registros ausentes, fragiliza o acompanhamento integral à saúde da criança e dificulta a comunicação entre os profissionais e serviços que participam da atenção à criança.

Nesse sentido, entende-se que é necessário promover capacitações para os profissionais, de modo a melhorar a qualidade dos registros na CSC, proporcionando que ela cumpra seu objetivo. Somado a isso, promover a sensibilização da família e dos profissionais sobre a importância da CSC como instrumento de vigilância da saúde infantil. 


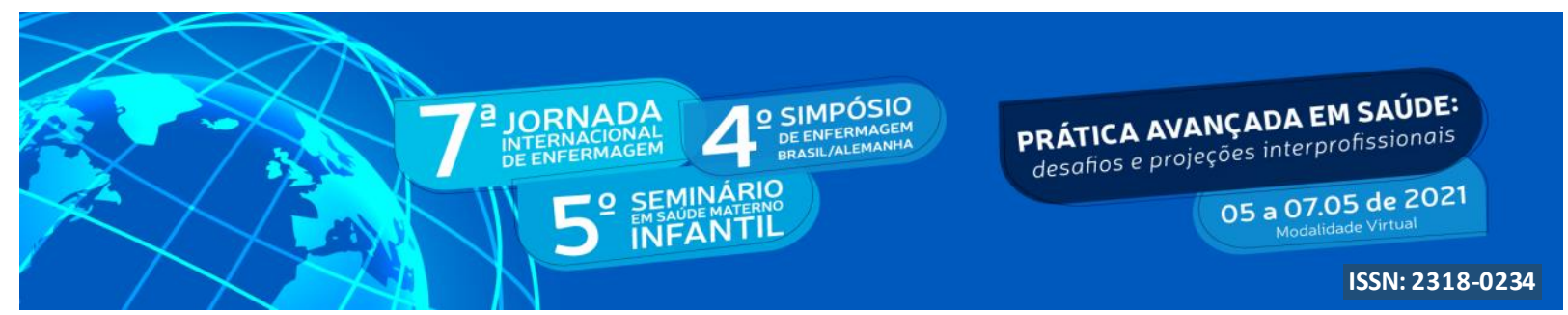

Quanto às limitações, entende-se que por ser um estudo documental, evidenciou-se o manejo inadequado, mas ficaram lacunas sobre o contexto que propicia essa inadequação. Sendo assim, sugere-se a realização de estudo qualitativo com profissionais da Atenção Primária em Saúde, de modo a elucidar a utilização inadequada da CSC, em especial para o acompanhamento do desenvolvimento infantil.

\section{REFERÊNCIAS}

ABUD, S.M.; GAIV A, M.A.M. Registro dos dados de crescimento e desenvolvimento na caderneta de saúde da criança. Rev Gaúcha Enferm. 2015 jun;36(2):97-105. Disponível em: <http://www.scielo.br/pdf/rgenf/v36n2/pt_1983-1447-rgenf-36-02-00097.pdf> Acesso em: 05 Fev. 2019. DOI: http://dx.doi.org/10.1590/19831447.2015.02.48427

ALMEIDA, A.C.; MENDES, L.C.; SADA, I.R.; RAMOS, E.G.; FONSECA, V.M.; PEIXOTO, M.V.M. Uso de instrumento de acompanhamento do crescimento e desenvolvimento da criança no Brasil - Revisão sistemática de literatura. Rev Paul Pediatr. 2015; 34(1):122-31. Disponível em: <http://www.scielo.br/pdf/rpp/v34n1/pt_0103-0582-rpp34-01-0122.pdf> Acesso em 05 Fev. 2019. http://dx.doi.org/10.1016/j.rppede.2015.12.002

AMORIM, L.P.; SENNA, M.I.B.; GOMES, V.E.; AMARAL, J.H.L.; VASCONCELOS, M.; SILVA, A.G. et al. Preenchimento da Caderneta de Saúde da Criança nos serviços de saúde em Belo Horizonte, Minas Gerais, Brasil. Rev Epidemiologia e Serviços de Saúde, 2018; 27(1):e201701116. Disponível em: <http://www.scielo.br/scielo.php?script=sci_arttext\&pid=S223796222018000100308\&lng=en\&nrm=iso>. Acesso em: 27 Jan. 2019. Epub Feb 15, 2018. http://dx.doi.org/10.5123/s1679-49742018000100016

ANDRADE, G. N.; REZENDE, T. M. R. L.; MADEIRA, A. M. F. Caderneta de Saúde da Criança: experiências dos profissionais da atenção primária à saúde. Rev Esc Enferm USP, São Paulo, v.48, n.5, p. 857-64, 2014. Disponível em:

<http://www.scielo.br/pdf/reeusp/v48n5/pt_0080-6234-reeusp-48-05-857.pdf> Acesso em: 20 Jul. 2018.

BRASIL. CONSELHO NACIONAL DE SAÚDE. Resolução nº 510 de 7 de abril de 2016.

Ministério da Saúde. Secretaria de Políticas de Saúde. Departamento de Atenção Básica. Saúde da criança: acompanhamento do crescimento e desenvolvimento infantil / Brasília: Ministério da Saúde, 2002

Ministério da Saúde. Portaria $n^{\circ}$ 964, de 23 de junho de 2005. Aprova a Resolução MERCOSUL/GMC no 04/05 e seu anexo intitulado "Informação Básica Comum para a Caderneta de Saúde da Criança". Diário Oficial da República Federativa do Brasil, Brasília 


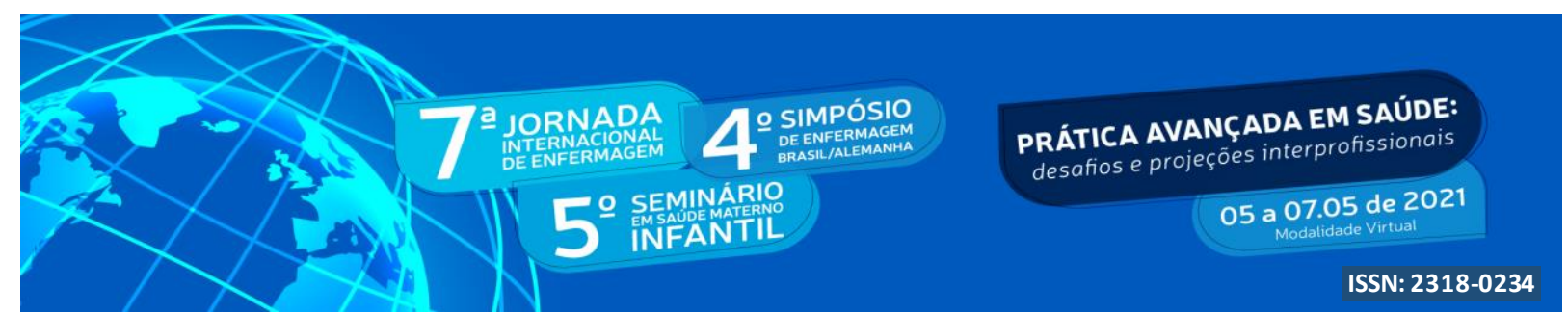

(DF), 2005a jun 24; Seção I, p. 74.

Ministério da Saúde. Secretaria de Atenção à Saúde. Departamento de Ações

Programáticas Estratégicas em Saúde. Área Técnica da Saúde da Criança e Aleitamento Materno. Manual para utilização da caderneta de saúde da criança.- Brasília: Ministério da Saúde, $2005 b$.

Ministério da Saúde. Secretaria de Atenção à Saúde. Área Técnica de Saúde da Criança e Aleitamento Materno. Gestões e gestores de políticas públicas de atenção à saúde da criança: 70 anos de história / Ministério da Saúde, Secretaria de Atenção à Saúde. Área Técnica de Saúde da Criança e Aleitamento Materno. - Brasília : Ministério da Saúde, 2011. 80 p. : il. - (Série I. História da Saúde)

Ministério da Saúde. Secretaria de Atenção à Saúde. Departamento de Atenção Básica. Saúde da criança : crescimento e desenvolvimento / Ministério da Saúde. Secretaria de Atenção à Saúde. Departamento de Atenção Básica. - Brasília : Ministério da Saúde, 2012. 272 p.: il. - (Cadernos de Atenção Básica, nº 33.

Ministério da Saúde. Portaria $\mathrm{n}^{\circ} 1.130$, de 05 de agosto de 2015. Institui a Política Nacional de Atenção Integral à Saúde da Criança (PNAISC) no âmbito do Sistema Único de Saúde (SUS). Diário Oficial da República Federativad o Brasil, Brasília (DF), 2015 ago 6; Seção I, p.37.

MONTEIRO, F.P.M.; ARAUJO, T.L.; XIMENES, L.B. e VIEIRA, N.F.C. Ações de promoção da saúde realizadas por enfermeiros na avaliação do crescimento e desenvolvimento infantil. Universidad de Concepción Concepción, Chile. Ciencia y Enfermería, vol. XX, núm. 1, abril, 2014, pp. 97-11Disponível em: <http://www.redalyc.org/pdf/3704/370441815009.pdf> Acesso em: 10 Mai. 2018

SANINE, P.R; CASTANHEIRA, E.R.L. Explorand o nexos entre construção social da criança e as práticas de saúde. História, Ciências, Saúde-Manguinhos, vol. 25, n 1, 2018, 199-215.

Casa de Oswaldo Cruz, Fundação Oswaldo Cruz. Disponível em: http://www.redalyc.org/articulo.oa?id=386155133012 Acesso em: 19 Jan. 2019. http://dx.doi.org/10.1590/S0104-59702018000100012

SILVA, F.B. e GAÍVA, M.A.M. Preenchimento da Caderneta de Saúde da criança: percepção dos profissionais. Cienc Cuid Saude, Vol 14, 2015. Disponível em :

$<$ https://scholar.google.com.br/scholar?hl=ptBR\&as_sdt=0\%2C5\&as_ylo=2014\&q=preenchi mento+da +caderneta+de+sa\%C3\%BAde+da+crian\%C3\%A7a\&btnG $\Rightarrow$ Acesso em: 09 Mai. 2018. 